Aim of the study: This study aimed to assess the efficacy of anthracycline-based $(A B)$ and non-anthracycline-based ( $n A B)$ adjuvant therapies in the human epidermal growth factor receptor 2 (HER2)-positive non-metastatic BC (nMBC) patients.

Material and methods: This retrospective study included women with HER2-positive BCs (stage I-III) treated with trastuzumab from 2008 to 2017. The patients were divided into two groups, including 196 patients in group $A B$ and 67 in group $n A B$.

Results: Cox's proportional hazard regression analysis showed no unfavourable predictors for five-year overall survival (OS) and disease-free survival (DFS) except for stage and hormone therapy. The OS rate was $67.9 \%$ in group $A B$ and $80.6 \%$ in group nAB $(p=0.630)$. The DFS rate was $61.6 \%$ in group $A B$ compared with $67.1 \%$ in group $\mathrm{nAB}(p=0.447)$.

Conclusions: The results showed no difference between the efficacies of $A B$ and $n A B$ regimens in HER2-positive $n M B C s$ in adjuvant setting. Therefore, selecting the $n A B$ regimen can reduce the serious damage caused by the $A B$ regimen.

Key words: breast cancer, HER2, trastuzumab, anthracycline, adjuvant therapy.

Contemp Oncol (Pozn) 2018; 22 (2): 108-112 DOI: https://doi.org/10.5114/wo.2018.77048

\section{The comparison of anthracycline- based and non-anthracycline-based regimens in adjuvant chemotherapy of HER2-positive non-metastatic breast cancers}

\author{
Safa Najafi ${ }^{1}$, Masoud Sadeghi ${ }^{2}$, Mohammad Reza Shajari ${ }^{1}$, \\ Fatemeh Abasvandi ${ }^{1}$, Kamran Mohebi ${ }^{1}$
}

${ }^{1}$ Breast Diseases Department, Breast Cancer Research Centre, Motamed Cancer Institute, ACECR, Tehran, Iran

${ }^{2}$ Medical Biology Research Centre, Kermanshah University of Medical Sciences, Kermanshah, Iran

\section{Introduction}

The most common cancer in women in the developed or developing countries is breast cancer (BC), with a peak in the age of 40-50 years in Asia [1]. The human epidermal growth factor receptor 2 (HER2) is overexpressed in approximately $15 \%-25 \%$ of invasive BCs and is associated with a high risk of disease recurrence and reduced survival $[2,3]$. Trastuzumab alone and in combination with chemotherapy has been identified to have a good safety record and to be active in advanced HER2-positive disease [4, 5]. Adjuvant systemic chemotherapy has significantly improved the prognosis of $B C$ patients [6]. Data from randomised trials including adjuvant therapy have reported that the risk of recurrence and death of disease is significantly reduced when adjuvant chemotherapy and/or hormonal therapy is added to the treatment [7]. Various factors have recently prompted a re-assessment of the role of non-anthracycline-based (nAB) regimens in the early stage of $\mathrm{BC}$ [8]. This debate has been triggered by recent data demonstrating the efficacy of $n A B$ regimens in various disease settings and highlighting the importance of anthracycline-related toxicity [8]. The benefits of anthracycline-based $(A B)$ regimens came with a number of clinically significant, long-term toxicities, in particular direct myocardial toxicity, which could lead to the later development of congestive cardiac failure and bone marrow damage resulting in leukaemia and myelodysplastic syndromes [9]. High histological grade, oestrogen receptor (OR)-negative, progesterone receptor (PR)-negative, or HER2-positive are risk factors involved in the recurrence, especially in the early recurrence, among which OR is the most significant prognostic factor involved in early recurrence [10].

The aim of this study was to evaluate the efficacy of $A B$ and $n A B$ adjuvant therapies in HER2-positive non-metastatic BC (nMBC) patients.

\section{Material and methods}

This retrospective study was approved by the Ethics Committee of Kermanshah University of Medical Sciences, Kermanshah, Iran. Patients referring to the Breast Cancer Research Centre, Tehran University of Medical Sciences, Tehran, Iran, during 2008-2017 were included in this study.

\section{Patients}

The patients were divided into two groups (196 patients were treated with $A B$ regimen [group $A B$ ] and 67 with $n A B$ regimen [group $n A B$ ]). The clin- 
icopathological and demographic factors were extracted for every patient based on the pathological and clinical reports. The median follow-up time was 42 months (range: 12-125 months). Of patients, $25 \%$ were treated with modified radical mastectomy and $75 \%$ were treated with breast conservation therapy. Radiotherapy was done based on the stage of disease, including 50 Gray (Gy) in 25 fractions plus 5 Gy in five fractions as a boost for the necessary items. Chemotherapy regimens were AC4T4 (cyclophosphamide-adriamycin [AC]) used every three weeks for four cycles, followed by paclitaxel ( $T$ ) given every three weeks for four cycles, or anthracycline-based regimens and TC (docetaxel and cyclophosphamide) given every three weeks for four cycles, or nAB regimen. Out of all patients, $15 \%$ had preoperative chemotherapy and $12 \%$ were inoperable or had inflammatory BC. All patients had Karnofsky Performance Score (KPS) of 0 and 1 . Only $0.5 \%$ patients had local recurrence and $99.5 \%$ had distant metastases. Out of all patients, $15 \%$ had neoadjuvant chemotherapy and $100 \%$ had adjuvant hormone therapy.

The inclusion criteria consisted of the female patients aged > 20 years; HER2-positive (HER2 $3+$ or HER2 $2+$ and fluorescence in situ hybridization (FISH) positivity) and non-metastatic patients (stage I-III). The exclusion criteria consisted of the patients with stage IV; the patients receiving Trastuzumab therapy as adjuvant therapy for less than one year, and the patients with the ejection fraction (EF) less than $45 \%$.

\section{Survival and recurrence}

Overall survival (OS) was defined as the time from the diagnosis to death by any cause, and disease-free survival (DFS) was defined as the time from the diagnosis to recurrence and/or metastasis, whichever was the earliest. OS and DFS were determined for five years. Breast tumour recurrence was defined as a comeback of cancer to the same place as the original (primary) tumour or to another place in the body. Distant metastasis was defined as recurrence at a distant site from the primary cancer.

\section{Statistical analyses}

The analysis was done by IBM SPSS (version 22) software (IBM Corp., Armonk, NY, USA). Chi-square test and t-test were used for the analysis of categorical and contin-

Table 1 . The comparison of baseline variables between the patients with anthracycline-based (AB) and non-anthracycline-based (nAB) chemotherapies

\begin{tabular}{|c|c|c|c|}
\hline & $\begin{array}{c}\text { Group AB } \\
n=196\end{array}$ & $\begin{array}{l}\text { Group nAB } \\
n=67\end{array}$ & $p$-value \\
\hline $\begin{array}{l}\text { Age (year), } n(\%) \\
\text { Mean } \pm S D \geq 50\end{array}$ & $\begin{array}{l}85(43.4) \\
47.8 \pm 11.6\end{array}$ & $\begin{array}{c}24(35.8) \\
46.2 \pm 10.8\end{array}$ & $\begin{array}{l}0.174 \\
0.325\end{array}$ \\
\hline $\begin{array}{l}\text { Type of pathology } \\
\text { IDC/DCIS/LC, } n(\%)\end{array}$ & $182(92.8) / 7(3.6) / 7(3.6)$ & $65(97) / 1(1.5) / 1(1.5)$ & 0.470 \\
\hline $\begin{array}{l}\text { Stage I/II/III, } \\
n(\%)\end{array}$ & 39 (19.9)/88 (44.9)/69 (35.2) & $27(40.3) / 26(38.8) / 14(20.9)$ & 0.003 \\
\hline $\begin{array}{l}\text { Lymph node metastasis } \\
\text { Yes, } n(\%) \\
\text { NO/N1/N2/N3 }\end{array}$ & $\begin{array}{c}132(67.3) \\
64 / 63 / 60 / 9\end{array}$ & $\begin{array}{c}28(41.8) \\
39 / 14 / 10 / 4\end{array}$ & $\begin{array}{c}<0.001 \\
0.002\end{array}$ \\
\hline $\begin{array}{l}\text { Tumour size }(\mathrm{cm}), n(\%) \\
\text { Mean } \pm \text { SD } \\
<2 / 2-5 />5\end{array}$ & $\begin{array}{c}3.2 \pm 1.6 \\
35(17.9) / 138(70.4) / 23(11.7)\end{array}$ & $\begin{array}{c}3.0 \pm 1.5 \\
13(19.4) / 49(73.1) / 5(7.5)\end{array}$ & $\begin{array}{l}0.463 \\
0.615\end{array}$ \\
\hline $\begin{array}{l}\text { Grade, } n(\%) \\
\mid / / I / I I\end{array}$ & $9(4.6) / 110(56.1) / 77(39.3)$ & $4(6) / 24(35.8) / 39(58.2)$ & 0.016 \\
\hline $\begin{array}{l}\text { Vascular invasion, } n(\%) \\
\text { Yes }\end{array}$ & $106(54.1)$ & $33(49.3)$ & 0.294 \\
\hline $\begin{array}{l}\text { Laterality, } n \text { (\%) } \\
\text { Right }\end{array}$ & $100(51)$ & 35 (52.2) & 0.488 \\
\hline $\begin{array}{l}\text { Ki67 index, \% }(n=207) \\
\text { Mean } \pm \text { SD } \\
\leq 20\end{array}$ & $\begin{array}{c}28.4 \pm 20.2 \\
64(44.1)\end{array}$ & $\begin{array}{c}29.7 \pm 18.4 \\
24(38.7)\end{array}$ & $\begin{array}{l}0.669 \\
0.285\end{array}$ \\
\hline $\begin{array}{l}\mathrm{ER}, n(\%) \\
\text { Positive }\end{array}$ & $119(60.7)$ & $44(65.7)$ & 0.284 \\
\hline $\begin{array}{l}\mathrm{PR}, n(\%) \\
\text { Positive }\end{array}$ & $101(51.5)$ & $37(55.2)$ & 0.352 \\
\hline $\begin{array}{l}\text { Radiotherapy, } n(\%) \\
\text { Yes }\end{array}$ & $167(85.2)$ & $50(74.6)$ & 0.040 \\
\hline $\begin{array}{l}\text { Hormone therapy, } n(\%) \\
\text { Yes }\end{array}$ & $133(67.9)$ & $40(59.7)$ & 0.143 \\
\hline
\end{tabular}

IDC - invasive ductal carcinoma; DCIS - ductal carcinoma in situ; LC - lobular carcinoma; N-number of lymph node; SD - standard deviation 
Variables

Chemotherapy group, $A B$ vs. $n A B$

Age, $\geq 50$ vs. $<50$ yrs

Type of pathology, IDC or DCIS vs. LC

Stage, 1 or 2 vs. 3

Lymph node metastasis, yes vs. no

Tumour size, $<2$ or $2-5$ vs. $>5 \mathrm{~cm}$

Grade, 1 or 2 vs. 3

Vascular invasion, yes vs. no

Laterality, right vs. left

Ki67 index, $\leq 20$ vs. $>20$

$E R$, positive vs. negative

$\mathrm{PR}$, positive vs. negative

Radiotherapy, yes vs. no

Hormone therapy, yes vs. no
Statistics for each study

Hazard ratio

1.001

0.731

0.974

2.149

2.173

1.106

1.376

1.722

0.976

0.839

0.454

1.055

1.186

2.655

1.130
Upper limit

$0.509 \quad 1.968$

$0.420 \quad 1.273$

0.673

1.120

1.410

4.124

5.786

2.029

0.603

0.799

2.369

0.907

0.553

3.270

1.724

1.485

0.474

0.143

0.400

0.562

1.040

0.955

$p$-value
0.998
0.268
0.889
0.021
0.120
0.745
0.250
0.097
0.933
0.547
0.180
0.914
0.654
0.041
0.153

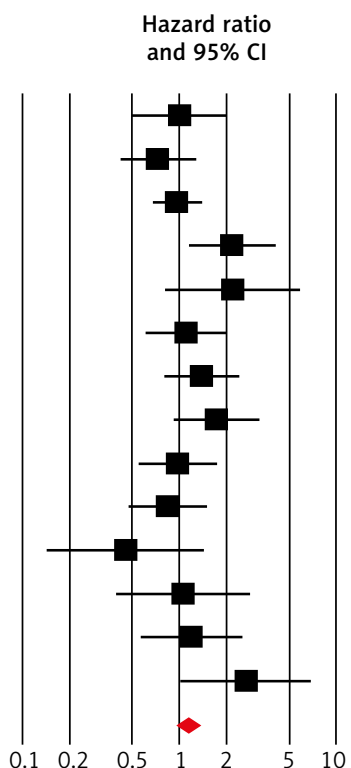

$\mathrm{Cl}$ - confidence interval; NA - not available; IDC - invasive ductal carcinoma; DCIS - ductal carcinoma in situ; LC - lobular carcinoma; AB - anthracycline-based chemotherapy; $\mathrm{nAB}$ - non-anthracycline-based chemotherapy; ER - estrogen receptor; PR - progesterone receptor

Fig. 1. Forest plot of the random-effect of hazard ratio of variables affecting the overall survival. Hazard ratios (HRs) are presented as the risk of the left-side category vs. the right-side category

Variables

Chemotherapy group, $A B$ vs. nAB

Age, $\geq 50$ vs. $<50$ yrs

Type of pathology, IDC or DCIS vs. LC

Stage, 1 or 2 vs. 3

Lymph node metastasis, yes vs. no

Tumour size, $<2$ or $2-5$ vs. $>5 \mathrm{~cm}$

Grade, 1 or 2 vs. 3

Vascular invasion, yes vs. no

Laterality, right vs. left

Ki67 index, $\leq 20$ vs. $>20$

$E R$, positive vs. negative

$\mathrm{PR}$, positive vs. negative

Radiotherapy, yes vs. no

Hormone therapy, yes vs. no
Statistics for each study

$\begin{array}{ccccc}\text { Hazard ratio } & \text { Lower limit } & \text { Upper limit } & z \text {-value } & p \text {-value } \\ 1.118 & 0.627 & 1.995 & 0.378 & 0.706 \\ 1.011 & 0.617 & 1.656 & 0.043 & 0.965 \\ 0.926 & 0.648 & 1.324 & -0.421 & 0.674 \\ 1.851 & 1.074 & 3.191 & 2.216 & 0.027 \\ 1.839 & 0.791 & 4.275 & 1.415 & 0.157 \\ 1.024 & 0.616 & 1.702 & 0.091 & 0.927 \\ 1.380 & 0.862 & 2.208 & 1.343 & 0.179 \\ 1.251 & 0.726 & 2.157 & 0.806 & 0.420 \\ 1.150 & 0.708 & 1.867 & 0.565 & 0.572 \\ 0.961 & 0.586 & 1.576 & -0.158 & 0.875 \\ 0.417 & 0.153 & 1.138 & -1.708 & 0.088 \\ 1.177 & 0.514 & 2.694 & 0.386 & 0.700 \\ 1.437 & 0.760 & 2.716 & 1.116 & 0.264 \\ 2.776 & 1.179 & 6.537 & 2.336 & 0.019 \\ 1.178 & 0.999 & 1.389 & 2.051 & 0.051\end{array}$

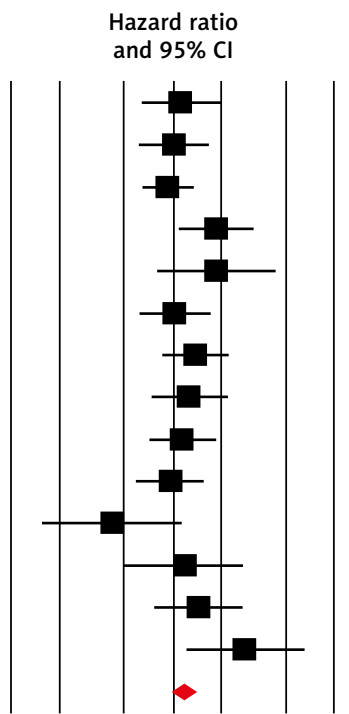

$\mathrm{Cl}$ - confidence interval; NA - not available; IDC - invasive ductal carcinoma; DCIS - ductal carcinoma in situ; LC - lobular carcinoma; AB - anthracycline based chemotherapy; $\mathrm{nAB}$ - non-anthracycline based chemotherapy; ER - estrogen receptor; PR - progesterone receptor

Fig. 2. Forest plot of the random-effect of hazard ratio of the variables affecting disease-free survival. Hazard ratios (HRs) presented as the risk of the left-side category vs. the right-side category

uous data, respectively. OS, DFS, and adverse events were the outcomes of this study. The comparison between OS and DFS in the two groups was performed by GraphPad Prism 5 software. The log-rank test was run to compare the Kaplan-Meier curves for OS and DFS. Also, Cox's proportional hazard regression analysis was used to evaluate the effects of various parameters on the primary analysis. A $p$-value (two-tailed) less than 0.05 showed a statistically significant difference. Confidence interval (CI) was 95\% in all analyses. A forest plot of the random-effects model was made by Comprehensive Meta-Analysis software version 2.0 (CMA 2.0) for hazard ratios (HRs).

\section{Results}

This study included 263 female patients with BC, 169 in group $A B$ and 67 age-matched in group $n A B$ (Table 1). 

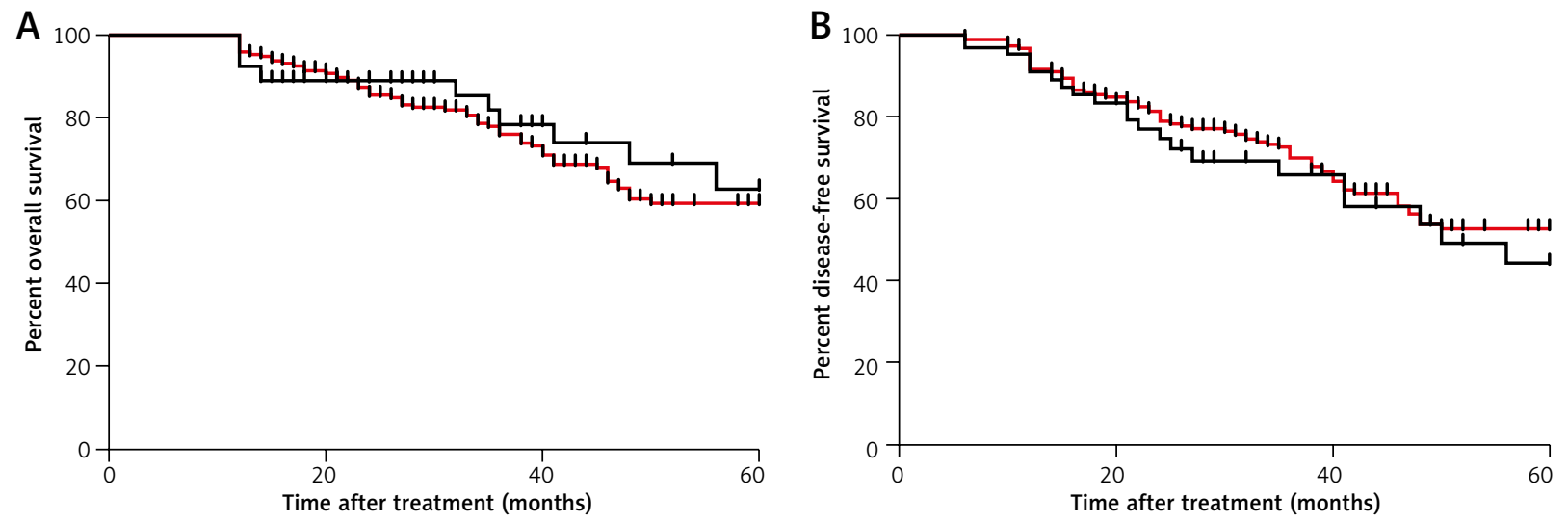

$\perp \mathrm{AB}$ - anthracycline-based chemotherapy group

$\perp \mathrm{nAB}$ - non-anthracycline-based chemotherapy group

Fig. 3. A) 5-year overall survival and B) 5-year disease-free survival for patients with breast cancer in two groups

Table 2. The comparison of adverse events between the patients with anthracycline-based (AB) and non-anthracycline-based (nAB) chemotherapies

$\begin{array}{lccc}\text { Adverse events } & \text { Group AB, } n=196 & \text { Group nAB, } n=67 & p \text {-value } \\ \text { Neutropaenia (grade 3-4) } & 10(5.1) & 1(1.4) & 0.232 \\ \text { Alopecia (grade 3-4) } & 194(98.5) & 47(70.1) & 0.001 \\ \text { Nausea and vomiting (grade 3-4) } & 10(5.1) & 1(1.4) & 0.232 \\ \text { Diarrhoea (grade 3-4) } & 2(1) & 2(3) & 0.279 \\ \text { Cardiovascular complications } & 7(3.6) & 1(1.4) & 0.407\end{array}$

Among the variables included, stage, lymph node metastasis, grade, and radiotherapy had different significant rates in the two groups $(p<0.05)$.

Cox's proportional hazard regression analysis was used to evaluate the effect of various parameters on the primary analysis. The hazard ratios of the variables affecting OS and DFS are shown in Figures 1 and 2, respectively. There were no unfavourable predictors for OS and DFS except for stage ( $p=0.021$ and $p=0.027$, respectively) and hormone therapy ( $p=0.041$ and $p=0.019$, respectively).

The OS rate and mean were $67.9 \%$ and 39.1 months in group $A B$ vs. $80.6 \%$ and 30.1 months in group $n A B$ ( $p=0.630$ ), respectively (Fig. 3 ). The DFS rate and mean were $61.6 \%$ and 36.9 months in group $A B$ vs. $67.1 \%$ and 28 months in group $\mathrm{nAB}(p=0.447)$. No significantly increased risk was seen in group $A B$, compared to group $n A B$, for either the five-year DFS (HR 0.82, 95\% Cl 0.49-1.37) or the five-year OS (HR 1.15, 95\% Cl 0.64-2.06).

Table 2 presents the comparison of adverse events between both groups. Among the mentioned adverse events, grade-3-4 alopecia was significantly higher in group $A B$ than group $\mathrm{nAB}(p<0.001)$.

\section{Discussion}

HER2 over-expression is an independent index for predicting poor prognosis and short DFS in BC patients. HER2-over-expressed patients are resistant to CMF regimen chemotherapy, but sensitive to $A B$ or anthracyclines plus taxanes regimen. HER2 expression can be taken as a marker for therapies in $B C$ [11]. The present study assessed the impact of two regimens ( $A B$ and $\mathrm{nAB}$ chemotherapies) on HER2-positive $n M B C$ patients in an adjuvant setting. The study showed that OS and DFS were similar in both chemotherapy regimens. Considering the results obtained from the randomised controlled trials, there are limitations to the evidence supporting the use of nAB regimens [8]. Many studies have indicated an association between anthracyclines and HER2 expression [12]. One study reported that the use of $A B$ chemotherapy declined, and the majority of patients with $B C$ were instead receiving taxane-based chemotherapy. However, the potential impact on patient outcomes is unknown. The $n A B$ regimens are likely to benefit patients in that the cardiac toxicity rates will decrease [13]. The $A B$ regimens are the most commonly used chemotherapies that have been shown to be associated with cognitive impairment and brain changes in clinical studies [14].

Several studies have reported that HER2-positive BC is particularly sensitive to anthracycline treatment [11, 15, 16]. Trastuzumab improves the overall survival of HER2-positive BC women but is associated with cardiotoxicity, especially when administered after anthracyclines [17]. Adjuvant chemotherapy with anthracyclines improves the disease-free and overall survival compared to $n A B$ adjuvant chemotherapy regimens in the treatment of early $B C$ [15]. A meta-analysis showed that the added benefits of adjuvant chemotherapy with anthracyclines appear to be confined to women who have overexpressed HER2 or amplified BCs [15]. 
Slamon et al. [2] reported that adding one year of ad juvant trastuzumab significantly improved DFS and OS among HER2-positive BC women. The risk-benefit ratio favoured the $\mathrm{nAB}$ regimen (docetaxel and carboplatin plus 52 weeks of trastuzumab) over the $A B$ regimen (doxorubicin and cyclophosphamide followed by docetaxel every three weeks) plus trastuzumab, given its similar efficacy, fewer acute toxic effects, and lower risks of cardiotoxicity and leukaemia. Almost all current adjuvant therapies incorporated the use of anthracyclines on the basis of the meta-analysis by the Early Breast Cancer Trialists' Group, which showed an improved efficacy of $A B$ chemotherapy regimens over regimens without anthracycline [18]. Analyses of HER2 status among approximately 5200 patients who participated in eight large, randomised adjuvant trials comparing $A B$ with $n A B$ chemotherapy regimens showed that only HER2-positive BC women derived an incremental benefit from anthracycline use $[19,20]$. The results of this study were similar to previous studies.

In conclusion, the results showed no difference between the efficacies of $A B$ and $n A B$ regimens in HER2-positive $n M B C s$ in an adjuvant setting. Therefore, $n A B$ regimen can reduce the serious damage caused by $A B$ regimen.

\section{The authors declare no conflict of interest.}

\section{References}

1. Payandeh M, Sadeghi M, Sadeghi E, Janbakhsh A. Is There any Concordance between of IHC with FISH in HER2-Positive Breast Cancer Patients? Int J Hematol Oncol Stem Cell Res 2017; 11: 43-48.

2. Slamon D, Eiermann W, Robert N, et al. Adjuvant trastuzumab in HER2-positive breast cancer. N Engl J Med 2011; 365: 1273-1283.

3. Gianni L, Dafni U, Gelber RD, et al. Treatment with trastuzumab for 1 year after adjuvant chemotherapy in patients with HER2-positive early breast cancer: a 4-year follow-up of a randomised controlled trial. Lancet Oncol 2011; 12: 236-244.

4. Pegram MD, Pienkowski T, Northfelt DW, et al. Results of two open-label, multicenter phase II studies of docetaxel, platinum salts, and trastuzumab in HER2-positive advanced breast cancer. J Natl Cancer Inst 2004; 96: 759-769.

5. Robert N, Leyland-Jones B, Asmar L, et al. Randomized phase III study of trastuzumab, paclitaxel, and carboplatin compared with trastuzumab and paclitaxel in women with HER-2-overexpressing metastatic breast cancer. J Clin Oncol 2006; 24: 2786-2792.

6. Chen X, Ye G, Zhang C, Li X, Shen K. Non-anthracycline-containing docetaxel and cyclophosphamide regimen is associated with sustained worse outcome compared with docetaxel, anthracycline and cyclophosphamide in neoadjuvant treatment of triple negative and HER2-positive breast cancer patients: updated follow-up data from NATT study. Chin J Cancer Res 2016; 28: 561-569.

7. Tack DK, Palmieri FM, Perez EA. Anthracycline vs nonanthracycline adjuvant therapy for breast cancer. Oncology (Williston Park) 2004; 18: 1367-1376.

8. de Boer RH, Chan A, Tran B, Wilcken N. Use of non-anthracycline regimens in early stage breast cancer in Australia. Asia Pac J Clin Oncol 2011; 7: 4-10.

9. Robson D, Verma S. Anthracyclines in early-stage breast cancer: is it the end of an era? Oncologist 2009; 14: 950-958.

10. Shahriari-Ahmadi A, Arabi M, Payandeh M, Sadeghi M. The recurrence frequency of breast cancer and its prognostic factors in Iranian patients. Int J Appl Basic Med Res 2017; 7: 40-43.

11. Zhang J, Liu Y. HER2 over-expression and response to different chemotherapy regimens in breast cancer. J Zhejiang Univ Sci B 2008; 9: 5-9.
12. Slamon DJ, Leyland-Jones B, Shak S, et al. Use of chemotherapy plus a monoclonal antibody against HER2 for metastatic breast cancer that overexpresses HER2. N Engl J Med 2001; 344: 783-792.

13. Giordano SH, Lin YL, Kuo YF, Hortobagyi GN, Goodwin JS. Decline in the use of anthracyclines for breast cancer. J Clin Oncol 2012; 30: 2232-2239.

14. Kesler SR, Blayney DW. Neurotoxic Effects of Anthracycline- vs Nonanthracycline-Based Chemotherapy on Cognition in Breast Cancer Survivors. JAMA Oncol 2016; 2: 185-192.

15. Gennari A, Sormani MP, Pronzato P, Puntoni M, Colozza M, Pfeffer $\mathrm{U}$, Bruzzi P. HER2 status and efficacy of adjuvant anthracyclines in early breast cancer: a pooled analysis of randomized trials. J Natl Cancer Inst 2008; 100: 14-20.

16. Cheang MC, Voduc KD, Tu D, et al. Responsiveness of intrinsic subtypes to adjuvant anthracycline substitution in the NCIC.CTG MA.5 randomized trial. Clin Cancer Res 2012; 18: 2402-2412.

17. Yu AF, Mukku RB, Verma S, Liu JE, Oeffinger KC, Steingart RM, Hudis CA, Dang CT. Cardiac safety of non-anthracycline trastuzumab-based therapy for HER2-positive breast cancer. Breast Cancer Res Treat 2017; 166: 241-247.

18. Early Breast Cancer Trialists' Collaborative Group. Polychemotherapy for early breast cancer: an overview of the randomised trials. Lancet 1998; 352: 930-942.

19. Pritchard KI, Shepherd LE, O'Malley FP, Andrulis IL, Tu D, Bramwell VH, Levine MN; National Cancer Institute of Canada Clinical Trials Group. HER2 and responsiveness of breast cancer to adjuvant chemotherapy. N Engl I Med 2006; 354: 2103-2111.

20. Dressler LG, Berry DA, Broadwater G, et al. Comparison of HER2 status by fluorescence in situ hybridization and immunohistochemistry to predict benefit from dose escalation of adjuvant doxorubicin-based therapy in node-positive breast cancer patients. J Clin Oncol 2005; 23: 4287-4297.

\section{Address for correspondence}

\section{Masoud Sadeghi}

Medical Biology Research Center

Kermanshah University of Medical Sciences

P.O. Box 67155-1616

Kermanshah, Iran

e-mail: sadeghi_mbrc@yahoo.com

Submitted: 8.03 .2018

Accepted: $\quad 10.05 .2018$ 